ÉGYPTE

monde arabe

\section{Égypte/Monde arabe}

$17 \mid 1994$

Soudan 2

\title{
Quelques aspects de l'évolution démographique du Soudan 1956-1993
}

Essai de critique et de mise en cohérence des données

\section{François Ireton}

\section{OpenEdition}

Journals

Édition électronique

URL : https://journals.openedition.org/ema/1445

DOI : $10.4000 /$ ema. 1445

ISSN : 2090-7273

Éditeur

CEDEJ - Centre d'études et de documentation économiques juridiques et sociales

Édition imprimée

Date de publication : 31 mars 1994

Pagination : 15-37

ISSN : 1110-5097

Référence électronique

François Ireton, «Quelques aspects de l'évolution démographique du Soudan 1956-1993 », Égypte/ Monde arabe [En ligne], 17 | 1994, mis en ligne le 08 juillet 2008, consulté le 07 juillet 2022. URL : http:// journals.openedition.org/ema/1445; DOI : https://doi.org/10.4000/ema.1445

Ce document a été généré automatiquement le 7 juillet 2022.

Tous droits réservés 


\section{Quelques aspects de l'évolution démographique du Soudan 1956-1993}

Essai de critique et de mise en cohérence des données

\section{François Ireton}

Depuis 1983, année du troisième recensement national de la population du Soudan (en abrégé RPS 83) - dont les premiers résultats n'ont été publiés qu'en 1986 - l'évolution démographique globale du pays n'était connue que par les estimations annuelles du Département de la statistique relevant du ministère soudanais des Finances et de la Planification économique, estimations reprises, moyennant corrections, par les grandes organisations internationales publiant des données sur la population par pays. $\mathrm{Si}$, même en l'absence d'un état civil pratiquant un enregistrement systématique des événements vitaux, les méthodes permettant des estimations des effectifs de population au niveau national produisent des résultats considérés comme relativement fiables dans un contexte écologique et sociopolitique stable (mais l'on verra que pour le Soudan, même en situation "normale» cette fiabilité est pour le moins relative), l'histoire de ce pays, durant les dix dernières années, a conjugué différents épisodes susceptibles de défier l'application de ces méthodes. Les deux plus marquants sont la reprise de la guerre du Sud en 1983 - guerre quasi ininterrompue depuis cette date - et, dans la zone sahélienne du pays dont l'agriculture était déjà depuis longtemps fragilisée, la survenue d'années de sécheresse dont la plus cruelle (1984) s'est traduite par la famine de 1985, qui a essentiellement frappé les provinces du Nord-Kordofan, du Nord-Darfour et de la mer Rouge. Outre une mortalité directe et indirecte dont l'ampleur a fait l'objet d'estimations nombreuses et contradictoires ${ }^{1}$, la guerre a provoqué plusieurs vagues d'exode vers les différents pays frontaliers du Sud-Soudan, souvent suivies de retours mais dans des proportions difficiles à évaluer. Par contre, les migrations dues à la famine de 1985 sont restées internes². Quant à l'ampleur des décès imputables à cette dernière, ainsi que la répartition par sexe et âge des victimes (dont dépend le degré d'incidence de la famine sur la natalité à venir), elles ont également donné lieu à des estimations très divergentes. Mais ces deux faits capitaux de l'histoire 
soudanaise des années 80 ne font pas qu'obérer la production d'estimations fiables d'effectifs nationaux de population; leurs conséquences (essentiellement les migrations internes) rendent plus délicates encore les estimations, durant la période intercensitaire, de la répartition de la population par région et milieu d'habitat.

2 Les premiers résultats du RPS 93, quelles qu'en soient les imperfections ( $c f$. infra),sont donc particulièrement bienvenus dans un contexte où manquent des bases sûres d'estimation des effectifs de population. Ceci d'autant que ce manque a permis des spéculations chiffrées ajoutant la touche démographique indispensable à la noirceur globale du tableau d'inspiration "catastrophiste " que des témoins autorisés tracent actuellement du Soudan, et qui se réduit trop souvent à quelques généralités hâtives ou à de grossières exagérations masquant mal un rapport condescendant à la réalité étudiée ; exagération comme, par exemple, celle qui consiste à attribuer au «Grand Khartoum » 4,5 millions d'habitants (soit un triplement de sa population en 10 ans !), alors que selon le RPS 93, la ville en compte entre 3 et 3,5 M (selon la définition qu'on en donne), ce qui correspond à un taux annuel moyen de croissance proche de $7 \%$ de la population résidante, à laquelle s'ajoutent dans la journée, comme dans toutes les grandes villes du monde, plusieurs centaines de milliers de personnes faisant la navette entre la métropole et l'extérieur. Il n'est pas question de nier la gravité de la « crise de longue durée » et à multiples facettes ${ }^{3}$ que connaît le Soudan depuis maintenant quinze ans, crise dont aucun « indicateur » ne laisse entrevoir la fin et dont les conséquences sociales, économiques et démographiques sont loin d'être toutes analysées (et même connues). L'utilisation de la rhétorique du chaos, véritable rituel d'évitement de l'analyse, introduit a priori la cohérence du pire dans bien des discours - « savants » ou non - tenus sur ce pays dont on n'hésite pas à annoncer presque chaque année, sans autre forme d'explications, que "plusieurs millions" de ses habitants y sont tout bonnement "menacés de famine ». Cette description convenue du chaos autorise à ne pas aller y regarder de plus près, cautionne toutes les formes d'interprétation fatalistes, banalisantes et anesthésiantes et favorise paradoxalement la non-intervention, comme l'a fort bien montré S. Brunei dans un ouvrage où il est souvent question du Soudan ${ }^{4}$.

3 La présente étude est née de l'insatisfaction que l'on peut éprouver face aux contradictions- présentées, moins sans doute par les données soudanaises elles-mêmes que par l'usage qui en est fait, par la substitution incessante des estimations des organismes internationaux aux données réelles et par la citation, pêle-mêle, de données corrigées et non corrigées tirées des recensements soudanais, sans souci minimum de mise en parallèle. Il s'agissait de reconsidérer le matériau brut issu des opérations de terrain afin de démêler ce qui était de l'ordre de l'estimation grossière, du long travail d'ajustement et de la donnée brute, de façon à repérer ce qui, ultérieurement, pourrait faire l'objet d'un retour aux sources statistiques et d'un nouveau travail de "première main" sur ces dernières, travail qui relève du démographe confirmé et non de ( « utilisateur de chiffres » qu'est l'auteur de ces lignes. Cette note est donc provisoire. Son but est de replacer, au bout de la série des données démographiques commençant au recensement de 1955/56, les effectifs de la population soudanaise (globale, ainsi que nord et sud-soudanaise) donnés par le RSP 93 ; on tentera de mener cet exercice de manière critique, c'est-à-dire en faisant le point sur les données, taux et indices disponibles et en en vérifiant la cohérence. On abordera au fur et à mesure, par ordre chronologique des recensements et périodes intercensitaires, les difficultés de leur estimation. 
4 Les résultats du RSP 93 ne sont encore que provisoires ${ }^{5}$ - les opérations de recensement proprement dites s'étant déroulées il y a moins d'un an - et ne concernent que les effectifs de population par sexe et le nombre de ménages, ceci pour l'ensemble des six États et des 51 muhâfazât formant le territoire du Nord-Soudan en avril 1993 (le système de division administrative a changé depuis cette date) ${ }^{6}$. Le RPS 93 est le quatrième recensement de population du Soudan. L'on dispose donc de quatre effectifs nationaux de population : le premier, au 1/1/56 (RSP 56), était de 10,263 millions (dorénavant $\mathrm{M}$ ) ; le second, au 3/4/73 (RSP 73) de 14,819 M; le troisième, au 14/2/83 (RPS 83) de 20,594 M et le quatrième, au 15/4/93, de 24,941 M (résultats provisoires). Les trois taux moyens annuels de croissance intercensitaire de la population globale résidante soudanaise, non corrigés, sont donc respectivement de 2,15\% (sur 17,25 ans), de 3,43\% (sur 9,25 ans) et de $1,89 \%$, et le taux moyen annuel de croissance sur les 37 ans et 3 mois qui séparent le premier recensement du quatrième s'établit à $2,41 \%$.

\section{Première période (1956-73) : une population stable et (presque) fermée?}

5 La progression du premier taux (2,15\%) au second (3,43\%) semble indiquer que le Soudan, à cette époque (1956-83) poursuivait son processus de transition démographique et se situait dans la première phase de cette dernière. Cette phase est dite de " transition de mortalité » : la mortalité y diminue, alors que la natalité, élevée, reste constante, voire s'accroît faiblement. Cependant, une telle augmentation du taux de croissance en treize ans et demi n'est guère possible, sauf à supposer que, durant la première période intercensitaire, le Soudan ait connu des surmortalités considérables (liées à des famines, des épidémies ou des guerres extrêmement meurtrières), ce qui n'a pas été le cas. Une telle progression - en admettant un taux de natalité constant supposerait en effet que le taux de mortalité ait diminué de 1 pour 1.000 par an, ce qui constituerait un record mondial de rapidité en matière de transition de mortalité. Mieux vaut admettre que ces taux sont erronés, c'est-à-dire que la couverture des recensements a varié de l'un à l'autre.

$6 \quad$ Les méthodes utilisées lors du RSP 56 ont été les suivantes : recensement exhaustif des 68 agglomérations urbaines de l'époque et, dans les zones rurales, sondage au 1/10e, par une procédure à trois degrés partie d'une cartographie exhaustive des sites habités, le tout suivi d'une enquête postcensitaire de contrôle qui a permis de corriger les sous et surdénombrements, selon les régions. Les effectifs définitifs donnés, qui intègrent ces ajustements, sont unanimement considérés comme fiables ${ }^{7}$. L'hypothèse peut donc déjà être faite d'une sous-estimation de la population au RPS 73, dont la "réestimation" relèverait le premier taux moyen de croissance et abaisserait le second (en considérant provisoirement les effectifs donnés comme fiables par le RPS 83).

7 L'on connaît les raisons qui auraient entraîné une sous-estimation de la population soudanaise au RSP 73 : ce dernier devant initialement avoir lieu en mai 1972 et les accords d'Addis Abeba ayant mis fin la même année à la première guerre du Sud, l'on a repoussé la date du recensement pour permettre aux réfugiés sud-soudanais partis dans les pays limitrophes de retourner chez eux. Mais en avril 1973, seule une faible partie d'entre eux avait regagné sa résidence habituelle ${ }^{8}$ et la région Sud était encore dans un état de grande perturbation, ce qui a rendu peu faciles les opérations de recensement et a favorisé le sous-dénombrement ; ce dernier expliquerait - beaucoup 
plus que les pertes directes et indirecte dues à la guerre elle-même - la croissance négative, entre 1956 et 1973, des régions du Haut-Nil et de l'Equatoria, et anormalement basse de celle du Bahr al-Ghazal. C'est donc essentiellement la population sud-soudanaise qui a été sous-estimée au RPS 73. Les effectifs donnés pour celle du Nord-Soudan ( $c f$. infra)sont considérés comme fiables; d'autant que, d'une part, suite à la constatation d'une diminution suspecte de la population nomade par rapport au RSP 56, cette dernière a été recomptée en avril-mai 1974 et ses effectifs réévalués, à la date du recensement, de $0,4 \mathrm{M}$ à 1,7 $\mathrm{M}$ et que, d'autre part, les effectifs de cotton pickers migrants saisonniers de la Gezira, dont on avait cru qu'ils seraient rentrés chez eux (dans les provinces du Nil Bleu, Nil Blanc, Nord-Darfour et Nord-Kordofan essentiellement) à la date du recensement, ont été réestimés (à 0,67 M) sur la base des registres du Gezira Board et une partie d'entre eux retrouvés et enquêtes. Les effectifs ainsi rectifiés ont été affectés d'un taux de correction global de $+5 \%$.

8 Sachant que le taux de croissance naturelle de la population soudanaise d'après les données du RPS 56, corrigées et estimées de façon extrêmement minutieuse par Demeny ${ }^{9}$, était déjà de 2,74 \% par an à cette date (avec un TBN de 48,7/1.000 et un TBM de 21,3/1.000) et qu'il était du même ordre en 1973 (avec probablement une quasistabilité de ses composantes) d'après les données corrigées du RPS $73^{10}$, la population du Soudan aurait été à cette date de 16,3 M en faisant l'hypothèse d'une population fermée, c'est-à-dire en admettant que les réfugiés du Sud ayant franchi les frontières étaient revenus, à la date du recensement, dans leur région d'origine (ce qu'ils ne firent que durant les quelques années qui suivirent). Ce problème mis à part, il semble bien que, durant la première période intercensitaire, sans avoir à proprement parler constitué un ensemble fermé, la population soudanaise n'a accueilli qu'un nombre restreint de réfugiés et d'étrangers :

1.26.000 Éthiopiens ont franchi la frontière en 1967, 5.000 Zaïrois sont restés au Sud-Soudan en 1973, 4.000 Ougandais y sont arrivés en 1972 et peu de Tchadiens s'étaient encore réfugiés au Dar Masalit (Nord-Darfour) à cette date (5.000 à peu près) ${ }^{11}$;

2. le nombre des étrangers semble cependant avoir été largement sous-estimé au RPS 73, maints d'entre eux s'étant déclarés de nationalité soudanaise : ils étaient 206.000 au RPS 56 contre 276.000 au RPS 73, soit un taux de croissance de seulement 1,6 \% (c'est au vu de cette sous-estimation que le nombre - au demeurant modeste - des réfugiés a été ajouté à celui des étrangers dans le calcul de l'ensemble des immigrés);

3. le nombre de Soudanais émigrés " provisoirement » à l'étranger était estimé à la même date à $150.000^{12}$, dont seulement 50.000 travailleurs partis pour la plupart dans la Péninsule arabique; à cette époque, la grande vague des migrations vers cette région n'avait pas encore commencé.

Si l'on s'en tient aux chiffres donnés ci-dessus, le solde migratoire externe soudanais pour la période 1956-73 (hors réfugiés sud-soudanais) serait légèrement négatif: 150.000 émigrés (en supposant leur nombre égal à zéro en 1956) contre 40.000 réfugiés venus de l'extérieur et un gain de 70.000 étrangers installés au Soudan, soit un solde de - 40000 . La sous-déclaration des étrangers comme tels fait qu'en réalité le solde était sans doute équilibré, voire légèrement positif.

10 Il ne serait probablement pas trop éloigné de la réalité de proposer un chiffre d'environ 16 M d'habitants pour le Soudan de 1973 :16,3 M donnés par la croissance naturelle, moins 300000 réfugiés non encore rentrés et, ce phénomène mis à part, un solde migratoire nul; ce qui correspond à un taux moyen de croissance intercensitaire 
1956-73 de 2,6 \%, au lieu de 2,5\%, avec un taux de croissance naturelle très voisin et des TBN et TBM restant au même niveau d'un bout à l'autre de la période (ce qui n'empêche sans doute pas les oscillations); en bref, une population stable et quasi fermée.

\section{Intermède onusien : explosion démographique ou panne de transition?}

11 Pour la même période (1955-75), il existe deux séries d'estimations différentes des taux moyens annuels d'accroissement de la population. Celle donnée dans le World Population Prospect (version 1992) de l'ONU propose, pour les quatre périodes quinquennales allant de 1955 à 1975, des taux respectifs de 1,91,2,03,2,29 et 2,89\%; appliqués aux fractions de période intercensitaire qui leur correspondent et «calés » sur la population au $1 / 1 / 1956$, cela donnerait une population de $15,035 \mathrm{M}$ en 1973 , assez proche du chiffre officiel tiré du RPS de la même année. Le taux de mortalité a subi, quant à lui, une décroissance régulière; par période de 5 ans de 1950-55 à 1970-75, le TBM est successivement passé par les valeurs suivantes : 27,1, 26,0, 24,8 et 23,0/1.000. Il n'en faudrait pas déduire pour autant que, les migrations en direction du (et vers le) Soudan étant données, dans le même tableau, comme nulles durant cette période, le TBN onusien puisse être obtenu en additionnant les taux de croissance globale (égal, donc, au taux de croissance naturelle) et de mortalité ! De mystérieux et malicieux résidus viennent en effet se glisser entre les lignes... Il vaut tout de même la peine de s'arrêter un instant sur cette démographie fiction qui fait totalement fi des données recueillies lors des recensements - et corrigées, pour ce qui est de celui de 1956, par une sommité de la recherche démographique ${ }^{13}$ - et d'essayer d'en trouver la genèse.

Une hypothèse vient à l'esprit : aux horloges onusiennes, en 1955, l'heure avait sonné pour le Soudan d'entamer - ou du moins d'accélérer - sa baisse de mortalité ; pour cela, il fallait que cette mortalité fût haute en début de période $(27,1 / 1.000$ contre les 21,3 de Demeny) et qu'elle descendît, alors que Demeny montre qu'elle était déjà relativement basse en 1956 et que les estimations de [Analytical Report du RSP 73 indiquent qu'elle marqua vraisemblablement un palier entre 1956 et 1972. Le TBN ne pouvant guère avoir varié et étant lui aussi élevé, puisque la transition de fécondité n'avait pas encore commencé, reste, d'après l'ONU, à 46-47/1.000, ce qui est plus conforme aux estimations de Demeny et des analystes du RSP 73. Mais, en bonne arithmétique, il aurait fallu qu'il grimpe à 52/1.000 en 1970-75 pour rendre compte de l'accélération du taux de croissance, passant de $2,3 \%$ à $2,9 \%$ en cinq ans, d'après l'ONU.

Si on la rapporte aux analyses de Demeny et des auteurs de ['Analytical Report, la série onusienne semble décrire une fiction, plaquant sur une évolution réelle, mais ignorée, une évolution passée observée ailleurs, et présente ainsi un Soudan menant sagement, sans à-coups et en son temps, la transition de mortalité réputée universelle; " sagement » n'est d'ailleurs pas le mot juste puisque, la natalité restant constante, un taux de croissance passant de 1,9 à 2,9\% en quinze ans suppose, là encore, un déclin de la mortalité tout à fait irréaliste. Or il n'en a rien été : en 1956, le Soudan avait déjà entamé un processus de transition démographique depuis plusieurs décennies, alors que les séries onusiennes laissent penser que le processus s'était amorcé depuis peu et se déroulait alors en surrégime. En fait, bien que l'on ne puisse pas savoir quel était le niveau de la mortalité soudanaise avant le démarrage de sa transition, le TBM onusien 
de 27,1/1.000 rapproche le Soudan de pays d'Afrique ${ }^{14}$ considérés comme n'ayant pas encore débuté leur transition démographique et dont le TBM se situe autour de 35/1.000. La période 1956-73 apparaît plutôt, pour le Soudan pris dans son ensemble, comme un palier, comme une panne, mais qui se produit à un stade déjà avancé du processus de transition et dont la surmortalité due à la guerre du Sud ne peut pas rendre compte à elle seule. Du même coup, la vision d'une croissance « exponentielle » se trouve disqualifiée.

Il serait bien sûr souhaitable d'expliquer cette " panne » et, pour cela, d'examiner les profils régionaux et ethniques de la transition démographique au Soudan ${ }^{15}$, mais cela dépasse le but de la présente étude (stagnation du système de santé, regain de certaines formes de morbidité ?). À s'en tenir à la désagrégation ethnique opérée par Demeny, il est clair qu'en 1956, Fur, Arabes, Nubiens, Beja et Nuba (tous habitant le Nord-Soudan) étaient engagés dans la transition de mortalité bien plus encore que la «moyenne nationale » ne pourrait le laisser croire (leurs TBM étaient respectivement de 16/1.000, 17 pour les deux suivants, 18 et 19 pour les deux derniers) alors que les TBM des ethnies du Sud se situaient autour de 31/1.000, ce qui semble signifier qu'elles s'y engageaient tout juste. Il n'en reste pas moins que, la province/métropole de Khartoum mise à part - dont le TBM décline légèrement de 1956 (17/1.000) à 1973 (15/1.000) - l'on retrouve, d'après les résultats corrigés du RPS $73^{16}$, des niveaux inchangés de mortalité dans les provinces du Nord, avec une légère tendance à la remontée dans certaines provinces. Les provinces du Sud, "en retard», ont quant à elles continué leur processus de transition de mortalité durant la période 1956-73, passant à un TBM moyen de 27/1.000 en 1973 (le rapport des masses démographiques de 1 à 3 entre le Sud et le Nord-Soudan explique que la faible remontée du TBM du Nord «compense » la baisse de mortalité du Sud, le taux national restant étale sur 17 ans). Au vu de ces profils régionaux ${ }^{17}$, il est loisible de se demander si les provinces du Nord n'avaient pas atteint une sorte de " plancher » de mortalité provisoirement incompressible, au vu des pratiques curatives et préventives en place, des niveaux nutritionnels, etc. C'est, bien sûr, du côté de l'histoire de la lutte contre la malaria et autres endémies, ainsi que de l'évolution de la mortalité infantile, qu'il faudrait aller voir.

Si un ultime doute pouvait subsister sur la qualité des corrections et estimations de Demeny et sur la plausibilité d'un si faible niveau national de mortalité en 1956, un simple coup d'oeil sur le tableau de Coale et Lorimer (signalé en note 14) permet d'observer qu'un tel niveau, autour de 1960, est celui des pays d'Afrique de l'Est frontaliers ou proches du Soudan (et du Sud-Soudan en particulier!) tels le Kenya (en $1962: 18 / 1.000$ ), l'Ouganda (en $1959: 22 / 1.000$ ), le Rwanda, le Burundi (même niveau) et, dans une moindre mesure, la Tanzanie. Ces pays d'Afrique de l'Est - dont le Soudan - se séparaient ainsi, à l'époque, de leurs homologues d'Afrique occidentale et centrale, où les TBM tournaient encore autour de 35/1.000 et pouvaient atteindre 38/1.000 (Mali, Angola).

16 Il serait injuste de ne pas mentionner l'existence d'une autre estimation de la croissance durant la période 1955-75, tirée de Demographic Estimates for Countries with a Population of 10 Millions or More: $1981^{18}$; cette publication donne, pour les quatre périodes quinquennales comprises dans cette période, les taux respectifs suivants : 2,7, 2,6, 2,7 et $3 \%$; ces taux, appliqués de la même façon que précédemment, "produisent » un effectif de $16,324 \mathrm{M}$ en avril 1973, ce qui n'est pas très éloigné du chiffre de population que l'on a proposé pour cette date. 


\section{La deuxième période intercensitaire (1973-83) : de faibles transformations}

17 Du même coup, en adoptant le chiffre de $16 \mathrm{M}$ d'habitants pour 1973 et en prenant celui de 21,6 M d'habitants en 1983 comme correspondant aux effectifs réels de la population soudanaise à cette date (il s'agit du chiffre "officiel» augmenté de $5 \%$ pour sousestimation normale), le taux moyen de croissance annuelle intercensitaire 1973-83 tombe à $3,1 \%$, contre les $3,43 \%$ calculés d'après les données non corrigées des RPS 73 et RPS $83^{19}$; ceci à supposer, bien sûr, que les données tirées de ce dernier recensement soient fiables. Ce troisième RSP a fait l'objet de l'ensemble des tests de cohérence interne des données qui avaient été appliqués à celles des deux premiers; les résultats de ces tests, bien qu'ils ne soient pas développés, comme cela avait été le cas en 1973, dans les pages du National Report formant l'introduction à la publication des tableaux statistiques nationaux, concluent à la fiabilité des données moyennant l'ajustement de $5 \%$. Cette fiabilité s'explique, entre autres raisons, par le fait que, à la date du RPS 83, ce qui devait devenir la '« deuxième guerre du Sud» n'avait pas encore éclaté. Il s'en fallut de quelques mois.

Rendre compte de ce taux moyen de 3,1\% de croissance globale annuelle, c'est là encore examiner la croissance naturelle et ses composantes, d'une part, et l'évolution des migrations internationales, d'autre part. En ce qui concerne la croissance naturelle, les niveaux de natalité et de mortalité caractérisant le début de la période (1973) ont été examinés plus haut; on étudiera ici successivement l'évolution, de cette date à 1983, de la mortalité des moins de cinq ans, puis de celle des adultes et enfin de l'évolution de l'indice synthétique de fécondité (ISF).

19 S'agissant de la mortalité des enfants de moins de 5 ans, quatre sources en donnent les estimations rétrospectives ${ }^{20}$ : les deux RPS de 1973 et 1983 pour l'ensemble du Soudan et les deux enquêtes sur la fécondité de 1979 (SFS) et de 1989/90 (SDHS) ${ }^{21}$ pour le seul Nord-Soudan. Au niveau national, d'après le RPS 83, la mortalité infanto-juvénile aurait enregistré une baisse durant la seconde période intercensitaire. C'est ce qui ressort de l'analyse de Althàe Hill ${ }^{22}$ qui fait à ce propos les commentaires suivants :

«... By using the [Coale Demeny] South model, it would be barely possible to reconcile the two censuses [RSP 73 et RSP 83] by hypothesising... a plateau up to the early 1970s, a sharp decline till the mid-1970s, and then a further plateau up to the early 1980s, but this appears very far-fetched. [...] The overall probability of dying by age 5 of about 150 around 1980 surprisingly indicates similar levels of mortality in north and south Sudan, unlike earlier data [...] This favorable shift for all Sudan could be attributed only to a rapide decline in childhood mortality in the south, presumably immediately after the peace accord in 1973. However, in view of the difficulties in reconciling data [...], it would be prudent to suspend judgment on both levels and trends in childhood mortality in southern and all Sudan until such time as further data become available on the South. »

Il semble cependant que cette baisse se soit concentrée au Sud du Soudan. En effet, s'agissant du Nord, les données tirées, pour les années 1965-1979, du SFS de 1979 et pour 1975-1985 du SDHS de 1989/90, et qui se raccordent très bien entre elles, montrent un quotient de mortalité entre 0 et 5 ans (5q0) quasi-constant oscillant autour de 145/1000 de 1965 à $1985^{23}$. C'est ainsi que la baisse révélée, au niveau national, par le RPS 83 (5q0 de 170/1.000 en 1970 et de 145/1.000 en 1980) proviendrait 
d'une amélioration principalement localisée dans le $\operatorname{Sud}^{24}$. Les taux de mortalité infantile (enfants de moins d'un an) de cette région étaient en effet ${ }^{25}$ extrêmement élevés en 1956, de l'ordre de 300 à 350/1.000. En tout état de cause, le déclin rapide de la mortalité infanto-juvénile du Sud serait intervenu relativement tôt, entre 1970 et 1975, années durant lesquelles le quotient de mortalité de 0 à 5 ans de l'ensemble du Soudan serait passé de 170 à 150/1.000 pour demeurer ensuite presque constant jusqu'en 1983.

21 Concernant la mortalité des adultes durant la seconde période intercensitaire et pour le seul Nord-Soudan, le SFS de 1979 donne des probabilités de survie de 25 à 60 ans pour chaque sexe, ainsi que leur évolution dans le temps ${ }^{26}$. Il en ressort que ces probabilités restent constantes pour les hommes jusque vers 1970 (à un niveau de $65 \%$ pour les hommes et de $73 \%$ pour les femmes) ; puis elles s'élèvent jusqu'en 1974, date à laquelle s'interrompent les données (à un niveau de $75 \%$ pour les hommes et de $79 \%$ pour les femmes). Comme le déclarent les auteurs du rapport, " extrapolating the trend suggests a steady increase in the chances of survival for both sexes by the time of the survey" (1979) ${ }^{27}$. Le RPS 83, quant à lui, n'a pris en compte, dans son questionnaire, que la suivie des mères, et permet de calculer, pour l'ensemble du Soudan, des données concernant des probabilités féminines de survie de 25 à 60 ans sur quatre ans de plus (jusqu'en 1978) : parties en 1970 d'un niveau plus faible (70 \%, puisqu'elles intègrent celles des femmes sud-soudanaises) que celui qui caractérisait à la même date celles des femmes du seul Nord-Soudan, ces probabilités confirment la croissance constatée dans cette dernière région et rejoignent en 1978 le niveau atteint en $1974^{28}$. L'on peut supposer que, mutatis mutandis (et bien que l'on ait affaire, en ce qui concerne les femmes, à une mortalité en partie liée aux maternités) la même tendance existe pour les hommes. S'est-elle poursuivie après 1978 ? Il semble que ce soit la cas, mais sans que l'on puisse connaître le rythme de ce déclin ${ }^{29}$.

De ces deux analyses concernant la mortalité des moins de cinq ans et des adultes de 25 à 60 ans, il ressort par ailleurs que la mortalité des seconds est plus basse que ce que laisserait supposer celle des premiers, au regard des schémas-types de mortalité par âge $^{30}$. Par ailleurs, les seules mesures disponibles et, de ce fait, utilisées ici (quotients de mortalité de 0 à 5 ans, probabilités de survie entre 25 et 60 ans, taux de mortalité par âge de 20 à 49 ans) laissent trop de "trous " pour qu'une véritable synthèse soit possible. Cependant, l'on peut avancer que la réduction - de $160^{31}$ à 150/1.000 - du quotient de mortalité de 0 à 5 ans durant les premières années de la période intercensitaire, suivie de sa stagnation durant la seconde, ainsi que l'augmentation des probabilités de survie des adultes de 25 à 60 ans durant l'ensemble de cette période, ont infléchi à la baisse le TBM national, mais n'ont pu le faire, sur dix ans, que de façon très modérée.

S'agissant de la fécondité, le Sudan Fertility Survey de 1979, qui n'a couvert que la population du Nord-Soudan, donne pour cette région et pour l'année 1978 un indice synthétique de fécondité (ISF) corrigé, calculé à partir des naissances survenues durant les douze mois ayant précédé l'enquête, de 6,9 enfants par femmes ${ }^{32}$, peu différent de l'ISF, estimé à partir des données du RSP 73, de 7,0 enfants en 1972. En 1979 donc, la transition de fécondité ne semblait toujours pas amorcée dans le Nord-Soudan. Si l'on s'en tient au scénario classique de la transition démographique, le Sud-Soudan étant en retard sur le Nord en matière de baisse de mortalité, il est peu probable qu'il ait vu, avant ce dernier, sa fécondité diminuer. Le SDHS de 1989/90 donne, toujours pour le 
Nord-Soudan, un ISF corrigé de 6,0 enfants en 1987, à comparer avec son homologue de 6,7 en $1976^{33}$. La transition de fécondité du Nord-Soudan s'est donc amorcée et poursuivie durant la décennie 80 . Les dernières années de la période intercensitaire 1973-83 en ont sans doute été les témoins. L'on peut ainsi penser que, durant cette période :

1. une certaine réduction de la mortalité s'est accompagnée d'une légère -réduction de la fécondité et de la natalité ;

2. la réduction de la première a été un peu plus marquée que celle de la seconde, qui n'intervient qu'à l'extrême fin de la période ;

3. le taux de croissance naturelle a donc été un peu plus élevé en fin de période qu'au début (on peut proposer un ordre de grandeur de 2,8 \%).

Cette deuxième période intercensitaire se caractériserait donc, au Nord-Soudan, par l'amorce de la transition de fécondité et la fin de la "panne » de transition de mortalité pour ce qui concerne les adultes; dans l'ensemble du pays, par la quasi-stagnation de la mortalité des moins de cinq ans; enfin, au Sud-Soudan, par la continuation de la transition de mortalité (pour les adultes), son interruption à la mi-période (pour les moins de 5 ans) et l'absence probable de démarrage de la baisse de la fécondité. Si l'on retient un 2,8\% de croissance naturelle en 1983, le taux de croissance moyen annuel serait donc de $2,75 \%$ pour la période (sur la base de $2,7 \%$ en 1973); une immigration nette externe de l'ordre de $0,35 \%$ de la population totale aurait donc annuellement contribué au taux de croissance moyen annuel global de 3,1\%. C'est cette contribution qu'il faut maintenant examiner.

Les migrations internationales vers le Soudan, de 1973 à 1983, concernent uniquement des réfugiés venus des pays frontaliers et, parmi eux, essentiellement des Éthiopiens, mais aussi des Ougandais et des Tchadiens. En 1980, ces réfugiés étaient, d'après les statistiques établies par le ministère des Affaires intérieures de Khartoum ${ }^{34}, 441.000$; parmi eux, les Ethiopiens étaient 390.000, dont au moins 250.000 Erythréens refoulés au Soudan après la contre-offensive de l'armée éthiopienne de 1978 (en 1977, la quasitotalité de l'Erythrée était sous le contrôle des indépendantistes erythréens) et 140.000 réfugiés venus d'autres régions de l'Ethiopie d'alors, dont 100.000 Tigréens et plus de 15.000 Oromos $^{35}$. En 1981, ces réfugiés étaient au nombre de 575.000 (dont 450.000 Ethiopiens, 100.000 Ougandais et 20.000 Tchadiens). En 1982, 250.000 réfugiés ougandais sont arrivés de la province du West Nile, livrée au pillage par l'armée après la réélection du président Milton Obote. On peut donc estimer qu'environ 825.000 personnes ayant trouvé refuge au Soudan y demeuraient en 1983 au moment du recensement. Leur nombre avait donc augmenté d'à peu près 775.000 depuis le RPS 73 . Enfin, les 300.000 réfugiés soudanais partis durant la précédente période intercensitaire et qui ne sont rentrés qu'après 1973, durant les trois ou quatre ans qui ont suivi les accords d'Addis Abeba, correspondaient en 1983 à une population d'environ 380.000 habitants (en adoptant un taux de croissance naturelle, propre au Sud, de l'ordre de $2,5 \%$ et en admettant qu'ils n'aient pas réémigré). C'est donc, en chiffres ronds, $1,15 \mathrm{M}$ d'immigrés (d'origine soudanaise ou étrangère) qui viendraient s'ajouter au solde naturel de la population soudanaise entre 1973 et 1983.

Durant la même période, l'intensité de l'émigration, essentiellement liée à la recherche d'un travail à l'étranger (pays de la péninsule arabique) s'est accrue considérablement. Les estimations dans ce domaine sont cependant extrêmement divergentes ${ }^{36}$; en fait, les premières années de la période intercensitaire constituent une phase de départs 
très nombreux, suivis d'une accalmie et d'une reprise des départs à partir de 1982. La comparaison des estimations (et de leurs justifications) rassemblées par G. Beaugé permet de penser qu'un chiffre moyen de.400.000 Soudanais résidant à l'étranger en 1973 serait plausible, sans que l'on puisse ici examiner les- modifications positives ou négatives qu'il conviendrait d'apporter à ce chiffre. Le nombre de Soudanais à l'étranger aurait ainsi augmenté de 250.000 en dix ans, chiffre qu'il faut défalquer des $1,15 \mathrm{M}$ d'immigrés (réfugiés) arrivés en dix ans pour obtenir le solde migratoire externe de la population soudanaise durant la deuxième période intercensitaire, solde qui est largement positif et atteint le chiffre d'environ 0,9 M.

En retranchant cet effectif de la population globale du pays en 1983 (21,6 M), l'on constate que, sans apport migratoire, celle-ci aurait atteint les 20,7 M (pour $16 \mathrm{M}$ d'habitants en 1973), ce qui correspond à un taux moyen annuel de croissance naturelle intercensitaire de $2,7 \%$, à rapprocher du taux de croissance globale de 3,1\%. De 1973 à 1983, l'immigration nette externe (un apport moyen annuel équivalent à $0,4 \%$ de la population totale) a donc contribué pour moins d'un huitième à la croissance globale de la population. La quasi-coïncidence des estimations, menées indépendamment, des composantes de la croissance naturelle, d'une part, et des migrations externes, d'autre part, n'en doit pas moins inciter à la prudence. Seul un travail de première main » d'ajustement des données du RPS 83 pourrait venir confirmer ou infirmer le «scénario » qui vient d'être restitué. Quelles que soient les modifications - sans doute légères - qui pourront y être apportées, il est patent que d'une période intercensitaire à l'autre, malgré de lentes modifications des composantes de la croissance naturelle, son intensité, au niveau national, a très peu varié en 27 ans et est resté de l'ordre de 2,7\% en moyenne annuelle.

\section{Troisième période (1983-93) : la prédominance des bouleversements migratoires}

Quant au passage du taux de croissance moyen intercensitaire corrigé de 3,1\%, pour la période 1973-83, à celui, non corrigé, de la troisième période intercensitaire (1,89\%), il supposerait un solde migratoire externe devenu fortement négatif et/ou une surmortalité «accidentelle » (guerre, épidémie, famine) considérable et/ou l'entrée de la population soudanaise, durant les dix dernières années, dans une phase de transition démographique au cours de laquelle son taux de croissance naturelle, pratiquement constant depuis 1956, se serait mis à décliner considérablement; dans ce dernier cas, le rythme annuel de réduction de la natalité aurait largement dépassé celui de la baisse de la mortalité, ce qui suppose qu'une grande partie des couples se soient engagés dans le contrôle de leur fécondité.

En admettant que la mortalité n'ait pas décliné durant la troisième période intercensitaire (cas le plus favorable - la surmortalité accidentelle mise à part - pour obtenir une baisse rapide du taux de croissance) et en retenant provisoirement l'hypothèse d'une population fermée, le passage d'un taux de croissance naturelle de $2,75 \%$ à $1,89 \%$ en dix ans supposerait un déclin moyen annuel de 0,9/1.000 du taux de natalité, ce qui serait considérable. Avant d'envisager les différentes possibilités expliquant une réduction du taux de croissance, il faut revenir aux résultats provisoires de 1993, qui sont le produit d'un premier comptage des individus à partir des 
questionnaires du recensement ${ }^{37}$ et envisager les conditions dans lesquelles se sont déroulées les opérations du RPS 93.

De ces conditions, l'on sait encore peu de chose dans le détail. Des anecdotes invérifiables courent sur des "quartiers entiers oubliés", mais elles sont rituelles, au Soudan comme dans d'autres pays - y compris ceux à tradition statistique bien établie ; l'on connaît toujours une famille « qui n'a pas vu passer les agents recenseurs » et l'on stigmatise l'absence de formation de ces derniers... Mais tout cela ne peut fournir une estimation sérieuse du taux d'omission. Comme toutes les grandes opérations d'État, un recensement national de population dépend de la situation politique prévalant dans le pays. Or, les événements se déroulant au Sud-Soudan n'ont pas permis que le recensement y soit réellement mené, alors que la population de ce qu'on nommait encore, en avril 1993, les six États ${ }^{38}$ du Nord-Soudan a été recensée de façon quasisystématique (ce qui n'implique pas l'exhaustivité).

31 Dans l'ensemble du Nord-Soudan, seule la muhâfaza de Kadugli, située dans la partie centrale-méridionale du Sud-Kordofan et où résid(ai)ent la plupart des Nuba - contre lesquels des opérations dites de jihâd (guerre sainte) ont été lancées - n'a pas été recensée entièrement, les conditions de sécurité minimales ne le permettant pas ${ }^{39}$. Seule la ville de Kadugli et le Rural Council nord ont été recensés systématiquement ; deux autres Rural Council ne l'ont été que très partiellement et un quatrième ne l'a pas été du tout. Dans les trois États du Sud, seule a été recensée la population des muhâfaza (ou parties de muhâfaza) qui étaient alors considérées par l'État comme présentant pour les agents recenseurs des conditions minimales de sécurité, c'est-à-dire, dans la zone de conflit, les régions qui étaient aux mains des troupes gouvernementales. Concernant ces trois États, l'on ne dispose donc que d'estimations de leur population. La base de la méthode employée pour ce faire est la suivante: les taux de variation (en fait ici de décroissance) 1983-1993 de la population des seules entités du Sud-Soudan recensées ont été appliqués aux effectifs totaux de population de 1983 des États dont elles font partie, puis corrigés. Mais, dans la mesure où rien ne garantit la représentativité de ces entités par rapport aux États auxquels elles appartiennent, il semble que ces estimations ne puissent pas être prises en compte. La fragilité de ces dernières et les très grandes difficultés que présentent le décompte des victimes directes et indirectes de la guerre, ainsi que celui des émigrés du Sud-Soudan résidant hors des frontières nationales au moment du recensement, font que le «dossier démographique » du SudSoudan, qui a déjà donné lieu à tant de débats, restera sans doute encore longtemps mystérieux, le RPS 93 n'étant susceptible d'y apporter une contribution fiable qu'une fois connus les effectifs d'émigrés survivants originaires du Sud et résidant dans l'un des États du Nord au moment du recensement ${ }^{40}$. Ainsi, compte tenu de cette absence de recensement systématique dans le Sud en 1993, il y a de fortes (mal)chances pour que le RPS 93 soit définitivement considéré comme un recensement du seul Nord-Soudan.

En prenant en compte les effectifs de population donnés pour les trois États du SudSoudan au RPS 93, l'évolution depuis 1983 serait la suivante : la population de l'État de Bahr al-Ghazal est estimée à 1,82 M (contre 2,27 en 1983, soit un taux moyen annuel de variation de - 2,16\%), celle de l'État du Haut-Nil serait passée de 1,594 M à 1,047 M (soit - 4,02 \% par an) et celle de l'État d'Equatoria de 1,408 M à 0,993 M (- 3,34\% par an). L'ensemble du Sud-Soudan aurait vu sa population globale décliner, de 5,27 M à 3,86 M, à un taux moyen de - $3 \%$ par an, que seraient censées expliquer à la fois la surmortalité due directement ou indirectement (famines) au conflit et la «surémigration » vers le 
Nord-Soudan et vers les pays frontaliers (pour raison de guerre également puisque l'émigration vers le Nord existait déjà, pour des raisons économiques, durant la période intercensitaire précédente). De façon nécessairement approximative, l'on peut simplement noter qu'avec un taux moyen annuel de croissance naturelle de 2,5\% (hors surmortalité, en supposant en moyenne pour la période 1983-93 des TBN et TBM sudsoudanais de respectivement 47/1.000 et 22/1.000), la population du Sud-Soudan aurait dû atteindre - dans l'hypothèse d'une population fermée - le chiffre de $6,8 \mathrm{M}$ d'habitants, soit $175 \%$ des effectifs estimés au RPS 93. Si l'on s'en tient à une estimation - minimale, d'après de nombreux auteurs ${ }^{41}$ - de $0,6 \mathrm{M}$ de décès en 10 ans, parmi la population du Sud, des suites directes ou indirectes de la guerre, la population (fermée) de cette région serait réduite à $6,35 \mathrm{M}^{42}$. C'est de ce chiffre qu'il faudrait retrancher les effectifs d'émigrés partis vers les pays frontaliers et vers le Nord-Soudan. Il ne semble pas qu'il y ait eu, vu les circonstances, d'immigrés étrangers ou soudanais venus vers le Sud durant cette période. Il y eut par contre des migrations-retours d'habitants du Sud réfugiés/deplacés franchissant les frontières nationales et régionales (entre le Nord et le Sud). Les 0,35 M d'Ougandais (cf. supra)présents au Sud-Soudan en 1983, devenus six ans plus tard 0,4 M, sont tous repartis dans leur pays d'origine entre 1987 et $1989^{43}$. Quant aux Sud-Soudanais réfugiés hors des frontières (pour la plupart en Ethiopie), leur nombre était estimé à 350.000 en $1989^{44}$ et vu la prolongation du conflit et les famines qui ont de nouveau frappé de larges zones du Sud ${ }^{45}$, il est probable qu'à la date du RPS 93 un demi-million de Sud-Soudanais avaient trouvé refuge au-delà des frontières. Ce sont donc 0,9 M de Sud-Soudanais et étrangers réfugiés au Sud-Soudan qui ont quitté ce dernier avant le RPS 93 et 5,45 M de Sud-Soudanais qui y auraient été comptés si une part importante de ces derniers n'avait quitté cette région pour le Nord-Soudan. En examinant l'évolution de la population de cette région, il sera peut-être possible de se faire une idée du nombre de Sud-Soudanais qui y étaient présents au RPS 93. Il est cependant à remarquer que le chiffre "modéré " souvent donné de 1,5 $\mathrm{M}$ de SudSoudanais venus - et restés jusqu'à présent - au Nord depuis la reprise des hostilités aboutirait à considérer que le chiffre de 3,86 M d'habitants donné pour le Sud-Soudan en 1993 est loin d'être absurde.

La population du Nord-Soudan est passée, en un peu plus de 37 ans (1956-93), de 7,48 M à $21,14 \mathrm{M}$ (résultats provisoires) et a donc un peu moins que triplé durant cette période, avec un taux moyen annuel de croissance de $2,83 \%$. Il est probable que, lors du dernier recensement comme des précédents, une certaine proportion de ménages et/ou d'individus n'a pas été recensée; en retenant un taux de sous-enregistrement au recensement de l'ordre de $5 \%$, c'est donc à $22,2 \mathrm{M}$ environ que pouvait s'élever la population de cette grande région en 1993 , pour $16,09 \mathrm{M}$ d'habitants en 1983 (également après correction de $+5 \%$ ). Le taux de croissance annuel moyen a donc été de $3,19 \%$ durant la dernière période intercensitaire. Il est, là encore, sensiblement supérieur au taux de croissance naturelle, qui était, on l'a vu, de l'ordre de 2,7 \% durant la période intercensitaire précédente, pour l'ensemble du Soudan. Les résultats provisoires du RPS 93 ne comportant aucune donnée permettant de calculer un taux de croissance naturelle à cette date, l'on reconduira, à titre purement indicatif, ce dernier taux pour la période 1983-93, ce qui donnerait, sans apports migratoires et sans surmortalité, un effectif de population de l'ordre de 21,14 M en 1993. Cependant, le Nord-Soudan a connu une famine très meurtrière en 1984-85, et une surmortalité directe et indirecte - due aux conflits qui se sont déroulés dans le Sud-Kordofan et le Darfour ${ }^{46}$. 

la famine ; 300.000 morts a semblé à de nombreux spécialistes constituer un chiffre sinistrement plausible et l'un des meilleurs d'entre eux, À. de Waal, a donné pour le seul Darfour, après examen minutieux des données démographiques disponibles et une longue présence sur le terrain, une surmortalité de famine en 1984-86 de 110.000 décès environ $^{47}$. D'un total estime de $0,4 \mathrm{M}$ de décès, dans le Nord-Soudan, pour l'ensemble de la surmortalité de famine et de conflit, l'on déduira 50.000 décès ${ }^{48}$. C'est donc à environ $20,8 \mathrm{M}^{49}$ que se seraient montés les effectifs de population du Nord-Soudan, sur la base de la seule croissance naturelle théorique diminuée des effets de la surmortalité. L'apport migratoire net serait donc de $1,4 \mathrm{M}$ à la date du RSP 93 , pour la troisième période intercensitaire. l'Erythrée, réfugiés dont les effectifs totaux étaient estimés à $0,84 \mathrm{M}$ en décembre 1990) et du Tchad (estimés à 110.000 à la même date) ${ }^{50}$. Au total, le Nord-Soudan comptait environ $1 \mathrm{M}$ de réfugiés étrangers en 1990, dont la quasi-totalité était encore présente en 1993, ceci malgré les changements politiques intervenus en Ethiopie (chute du régime de Mengistu) et en Erythrée (accession à l'Indépendance). Cet effectif dépasse de 530.000 celui des réfugiés qui y étaient présents en 1983 (Tchadiens et Ethiopiens y étaient 470.000). La différence subsistant entre l'immigration nette totale de 1983 à $1993(1,4 \mathrm{M})$ et cet apport de réfugiés étrangers durant la même période se monte à $0,87 \mathrm{M}$. Le nombre de déplacés-réfugiés venus du Sud-Soudan sera donc équivalent à ce chiffre, augmenté ou diminué, selon qu'il sera positif ou négatif, du solde externe des Soudanais du Nord partis travailler à l'étranger, avec ou sans leur famille.

\section{Conclusion}

On arrêtera là l'exercice, aucune donnée crédible récente n'ayant pu être consultée sur le nombre d'émigrés soudanais à l'étranger. L'on peut seulement observer que l'effectif hypothétique de 1,5 M de Sud-Soudanais arrivés et restés au Nord-Soudan depuis 1983 impliquerait arithmétiquement, sur la troisième période intercensitaire, un solde migratoire externe de Soudanais à l'étranger (travailleurs et leurs familles) de - 0,63 M, ce qui signifie qu'en 1993 on compterait environ un million de Soudanais à l'étranger (non comptés les enfants d'émigrés qui y sont nés), puisque leur effectif était déjà de $0,4 \mathrm{M}$ en 1983. Malgré les retours qui ont fait suite à la guerre du Golfe, ce chiffre ne peut être considéré comme impossible ${ }^{51}$.

En retenant ainsi 4,2 $\mathrm{M}$ d'habitants au Sud-Soudan (l'effectif donné plus haut réévalué de $5 \%$ ) et 22,2 M d'habitants au Nord, la population présente au Soudan en avril 1993 se monterait donc à $26,4 \mathrm{M}$, soit un taux de croissance moyen annuel intercensitaire de seulement $2 \%$, ce qui semble faible. Mais il ne faut pas oublier que conflits et famines ont fait un minimum d'un million de morts en dix ans, soit une surmortalité de 0,8 M durant la période, et qu'en comptant les réfugiés ougandais et sud-soudanais ayant (re)passé la frontière, ainsi que le chiffre hypothétique proposé pour l'émigration nette de travail-, l'on atteint le million et demi d'émigrés sortis du territoire soudanais durant la période de référence, sans retours définitifs durant cette dernière. La perte globale, hors décès attendus, s'élèverait donc à au moins $2,3 \mathrm{M}$, faiblement compensée par l'arrivée des $0,5 \mathrm{M}$ de réfugiés éthiopo-érythréens et tchadiens. L'absence de l'ensemble de ces phénomènes de départ comme d'arrivée (dont le solde est de 1,8 M)

Égypte/Monde arabe, 17 | 1994 
aurait donc assuré au Soudan une population de 28,2 M en 1993 (chiffre... très proche de l'estimation, à la même date, de la population soudanaise par le World Population Prospect de l'ONU, édition 1992).

Ainsi, durant la troisième période intercensitaire, des phénomènes écologicoéconomiques et politiques relevant, au moins dans leurs manifestations, de la courte durée, auront été déterminants, par la surmortalité et les migrations qu'ils ont engendrées, dans l'évolution démographique du Soudan, modifiant ainsi les tendances que le paradigme de la transition démographique pouvait laisser prévoir. Cependant, seule une étude méthodique et critique des résultats définitifs du RPS 93 pourra apporter, en particulier sur le nombre de natifs du Sud résidant dans le Nord ainsi que sur la fécondité et la mortalité récentes, des informations qui viendront préciser et sans doute modifier les modèles - en partie descriptifs, en partie hypothétiques - esquissés ici ${ }^{52}$; ceux-ci n'avaient pour but que de proposer à la discussion des ordres de grandeur, dans un domaine où la diffusion et l'utilisation de chiffres sont rien moins qu'innocentes et où les manieurs de chiffres sont parfois pris de la «folie des grandeurs », travers auquel on n'est pas sûr d'avoir totalement échappé.

\section{NOTES}

1. Voir, entre autres références, l'article de S. al-D.al-SHAZALI : « Le coût humain et social de la guerre civile ", Égypte/Monde Arabe 15-16, pp. 153-173.

2. Des habitants du Nord-Darfour ont cependant fui vers le Tchad et des pasteurs de la province de la mer Rouge se sont réfugiés en Erythrée. Mais il ne s'est agi que de migrations temporaires et, dans le deuxième cas d'un accroissement des effectifs de migrations saisonnières qui ne concernent habituellement que dès Beni Amer.

3. Le dossier du numéro double d'EMA 15-16, dossier intitulé précisément «Les crises soudanaises dans les années 80 ", en a décrit de nombreux aspects.

4. BRUNEL S., Une tragédie banalisée, la faim dans le monde, Paris, Hachette, 1991 (collection «Pluriel, intervention »).

5. Ils sont édités dans une brochure intitulée Population Census of Sudan 1993, Provisional Results, Census Office, Khartoum, Central Bureau of Statistics, juillet 1993.

6. C'est grâce à l'amicale recommandation de S. al-D. al-Shazali, enseignant et chercheur au DSRC de l'Université de Khartoum, que j'ai pu entrer en contact avec des responsables du RSP 93. Le document technique décrivant les méthodes et opérations du recensement étant encore à paraître, un certain nombre d'informations indispensables à l'interprétation des résultats ont été fournies par K. E. Vaidyanathan, expert-démographe de l'UNFPA, Chief Technical Adviser du Soudan Census Project, dont l'accueil chaleureux et la grande expérience ont été précieux. Que tous deux soit ici vivement remerciés.

7. Il y a $95 \%$ de chances pour que la population réelle du Soudan au RPS 56 ait été comprise entre 10,191 M et 10,335 M d'habitants (cf. 1956 Census Methods Report, vol. 1, H.Q. Council of Ministers, Republic of the Sudan, Department of Statistics, Population Census Office, Khartoum, p 197).

8. En octobre 1973, soit six mois après le RGP 73, seulement 150.000 réfugiés sud-soudanais avaient regagné leur région d'origine (d'après la Relief Resettlement and Repatriation 
Commission), sur un total l'ayant quitté entre 1956 et 1973 estimé autour de 500.000 (bien évidemment, une proportion non négligeable est décédée en exil).

9. Dans un article intitulé «The Demography of the Sudan : an Analysis of the 1955/56 Census » figurant dans le volume édité par W. Brass et A. J. Coale, The Demography of Tropical Africa, Princeton University Press, 1968, pp. 466-51.

10. Department of statistics, 1973 Census Analytical Report, vol. 2, Population Dynamics, Ministry of National Planning, Khartoum, 1980, p. 254.

11. Tous ces chiffres doivent bien sûr n'être pris que comme des ordres de grandeur et sont tirés de OMARA A. A., « Socio-economic and Demographic Effects of Refugees on Sudanese Society » in Aspects of Population Change and Development in the Sudan, Proceedings of the Second National Population Conference, 22-28 April 1982 (A.A.M. Farah, O. Al-M. Nur, T. al-A. al-Dawi, eds.) The Sudan National Population Committee, The Economic and Social Research Council, The National Council for Research, Khartoum, s. d., pp. 75-186.

12. Department of Statistics, 1980 , rapport cité en note 12 , chapitre 5 , portant sur les migrations internes et externes.

13. Précisons que l'analyse critique impressionnante des résultats du RPS 56 est menée par Demeny selon une double désagrégation de la population en ethnies et en régions (qui s'avèrent ainsi avoir des fécondités et des mortalités très différentes, une fois neutralisés les différentiels de sous-déclaration des naissances et des décès intervenus dans l'année ayant précédé le recensement) et que la correction des taux fait appel à la théorie des populations stables et aux tables types régionales de mortalité élaborées par lui et A. J. Coale (dans Regional Model Life Tables and Stable Populations, Princeton University Press, 1966).

14. On sait que le TBM (comme le TBN) ne sont pas toujours de bons indicateurs comparatifs, vu leur sensiblrté à la structure d'âges ; mais, s'agissant des pays africains, cette structure est peu différente d'un pays à l'autre. De plus, l'on ne dispose pas à cette époque, pour la mortalité soudanaise, d'autres indicateurs concernant l'ensemble de la population et susceptibles de servir à la comparaison avec d'autres pays d'Afrique (l'on ne possède que des probabilités de décès avant l'âge de cinq ans, $c f$. infra) ; pour les TBM, on se réfère ici au tableau 4.2 de l'article de A. J. Coale et F. Lorimer, "Summary of Estimates of Fertility and Mortality », pp. 157-161 de l'ouvrage cité en note 9.

15. Des matériaux considérables permettant d'esquisser ces profils ont été réunis par G. À. Balaoman dans son ouvrage stimulant, Peoples and Economics in the Sudan 1886-1956 (Havard University Center for Population Studies, Cambridge, Ma., 1976, 2e éd. rev. et augm. 1981), au chapitre II en particulier, intitulé » Population changes in the Sudan : 1899-1956 (pp. 53-154).

16. 1956 Census Analytical Report, vol. 2, chapitre IV, "Mortality» et M. K. Rizigalla et K. V. Ramachandran, " Mortality Levels and Differentiate in the Sudan ", in Aspects of Population Change and Development in the Sudan, Proceedings of the Second National Population Conference, op. cit. en note 13, pp. 57-68.

17. L'opposition géographique Nord/Sud primant nettement sur le facteur ethnique dans la détermination des niveaux de mortalité ; ce qui n'était pas le cas en 1956 en ce qui concerne la fécondité, les Zandé, par exemple, ayant une fécondité beaucoup plus faible que les autres populations du Sud.

18. Publié par le US Department of Commerce, Bureau of the Census, Washington, DC, 1981. Référence explicite est faite, dans cette publication, aux estimations de Demeny.

19. Population census office, Population and Housing Census of the Sudan 1983, National Report, Department of Statistics, Ministry of Finance and Economic Planning, Khartoum, 1989.

20. En interrogeant les femmes - classées selon l'âge - sur le nombre d'enfants qu'elles ont eus et le nombre de survivants parmi eux, l'on peut, lors d'une enquête à passage unique, reconstituer la mortalité infanto-juvénile (i.e. de zéro à cinq ans) des quinze à vingt dernières années.

Égypte/Monde arabe, 17 | 1994 
21. Voir Sudan Ministry of National Planning, The Sudan Fertility Survey 1979, Principal Report (2 vol.), Department of Statistics, Khartoum, and WFS (World Fertility Survey), International Statistical Institute, 1982, et Sudan Ministry of Economic and National Planning, Sudan Demographic and Health Survey 1989/90, Department of Statistics and Institute for Resource Development/Macro International, Inc., Columbia, Maryland, USA, 1991.

22. Voir sa contribution : Trends in Childhood Mortality » in Demographic Change in Sub-Saharian Africa (Population Dynamics of Sub-Saharian Africa Program), KarenÀ. Fopte, Kenneth H. Hill and Linda G. Martin Editors, National Academy Press, Washington, DC, 1993, pp. 153-217.

23. Les $5 q 0$ référant aux données du RPS 73, du RSP 83, du SFS et du SDHS sont tous basés sur les probabilités de décès de 0 à 5 ans calculées d'après le modèle Sud de Coale et Demeny. Les 5 q0 d'après RPS 73 et RSP 83 sont tirés de Effects of Health Programs on Child Mortality In Sub-Saharian Africa, Douglas C. Ewbank and James N. Gribbles Editors, National Academy Press, Washington, DC, 1993, tableau 2-1 p. 14.

24. S'il y a une forte tendance à la baisse du $5 q 0$ dans le Sud/liée sans doute en grande partie à la fin de la première guerre du Sud, il semble qu'elle ne puisse être en fait aussi importante que ce que les informations citées ici révèlent ; admettre en effet pour la période 1970-1980 la constance du 5q0 du Nord à 145/1.000 et un 5q0 de l'ensemble du Soudan à 170/1.000 en 1970-75 suppose un $5 q 0$ du Sud à 225/1.000 durant cette période quinquennale, vu le poids démographique de cette région dans l'ensemble national; par ailleurs, admettre un $5 q 0$ de l'ensemble du Soudan à 150/1.000 en 1975-80 suppose un 5q0 du Sud à 165/1.000; une baisse aussi rapide est peu probable.

25. Estimés par Demeny d'après le modèle Est, art. cit., p. 488.

26. Probabilités mesurées par la méthode dite de survie des ascendants (pères et/ou mères) qui est considérée comme l'une des plus fiables et calculées d'après les données du RPS 73 (NordSoudan seulement) et du SFS de 1979.

27. Toutes ces données figurent aux pages 75 et 76 du rapport du SFS (op. cit.).

28. Données extraites d'al-Tigani al-Taher et Saleh H. Abu al-Yaman, «Mortality levels and differentials » in Population in Sudan, Population Census Office, Department of Statistics, Ministry of Finance and Economic Planning, Khartoum, 1991.

29. Le SDHS 1989/90 fournit bien une estimation des taux de mortalité par âge quinquennal et par sexe, entre 15 et 49 ans et pour la période 1976-89, basée sur la méthode, malheureusement peu fiable, de la survie des frères et sœurs (SDHS Report, tableau 9.2, p.111). Dans le même tableau, une comparaison est faite - pour les femmes - entre ces taux et ceux calculés, pour la période 1973-76 et sur la base du RPS 83, par la méthode de la survie des mères; on constate, pour les tranches d'âge comprises entre 20 et 49 ans, une légère réduction de la mortalité.

30. Concernant le seul Nord-Soudan, la mortalité infantile (moins d'un an) étant de 100/1.000, l'on attendrait, selon la table de mortalité « africaine » de Brass, une probabilité de survie de 25 à 60 ans de l'ordre de 65 \%, largement dépassée ici (cf. SFS Principal Report, vol. 1, p. 75).

31. Niveau sans doute atteint vers 1973 .

32. L'ISF (SFS, 1979) sur 12 mois et non corrigé, est de 6,23 ; l'ISF, à la même date et lui aussi non corrigé, sur les cinq années ayant précédé l'enquête, est de 6,02 (corrigé, il est de 6,7) ; l'ISF sur douze mois, non corrigé et calculé d'après les données du RPS 73, était de 5,24 pour le NordSoudan et de 5,44 pour l'ensemble du Soudan (voir SFS, vol. 1, p. 61 et RSP 73, Analytical Report, vol. 2, chapitre 3, Fecundity).Tous les ISF corrigés l'on été d'après la méthode dite $\mathrm{P} / \mathrm{F}$ ratios de Brass (à partir du groupe d'âge de 20-34 ans).

33. L'ISF est donné non corrigé dans le SDHS Report (p. 27, tableau 3.3); il est de 4,96. Il a été corrigé à partir des groupe d'âges féminins de 20-34 ans (la correction à partir des seuls 20-30 ans donne un ISF de 5.64). Les années 1976 et 1987 correspondent aux années médianes des deux périodes quinquennales prises en compte pour le calcul de ces IFS. 
34. The Office of the Comissionner for Réfugiées (COR), statistiques extraites de À. À. Omara, article cité en note 11, pp. 178-79 (ces chiffres ne peuvent être qu'indicatifs).

35. Voir MARCHAL R., "Le Soudan terre d'asile ", in Le Soudan contemporain, sous la direction de M. Lavergne, Karthala/CERMOC, Paris, 1989, p. 577-579.

36. Voir BEAUGE G., "L'émigration soudanaise vers les pays producteurs de pétrole ", in Le Soudan contemporain (cité en note précédente), pp. 550-555.

37. Des questionnaires «longs" (avec questions concernant la fécondité, la mortalité et les migrations) ont été administrés à la totalité des ménages urbains et à $10 \%$ des ménages ruraux, des questionnaires courts aux $90 \%$ de ménages ruraux restants.

38. Ces six États de 1993 gardaient, à quelques modifications près, les limites qu'avaient les six provinces du Nord-Soudan en 1955-56.

39. D'après le listing des seconds résultats provisoires (début mars 1994), 162.000 habitants ont été réellement comptés dans la muhâfaza et l'estimation du nombre réel d'habitants donne un effectif de 320.000 habitants, correspondant, comme pour les muhâfaza proches d'al-Salam et de Diling, à un léger déclin de la population depuis 1983 (l'Area Council de Kadugli comptait alors 340.000 habitants).

40. Les tableaux de migration depuis la naissance seront, semble-t-il, consultables d'ici un an, une forte volonté existant au Census Office de hâter au maximum le traitement des données recueillies. Il n'y a dans le questionnaire du RPS 93, pas plus que dans les précédents, de question concernant la résidence immédiatement antérieure à celle occupée lors du recensement.

41. Voir AL-SHAZALI S. al-D., art. cit. en note 1, p. 161. L'auteur donne une estimation d'un demimillion en 1991. Il est à noter que les chiffres de décès dus au conflit donnent malheureusement lieu à de grossières sous-estimations des victimes civiles du côté de l'État soudanais et à une surenchère de l'autre : on a été jusqu'à parler de $2 \mathrm{M}$ de morts et de... $3 \mathrm{M}$ de réfugiés dans le Nord (confondant déplacés à l'intérieur du Sud et réfugiés/déplacés dans le Nord), ce qui signifierait qu'il n'y a pratiquement plus personne dans le Sud; il est vrai que d'autres parlaient aussi, en 1990, de $8 \mathrm{M}$ d'originaires du Sud, présents ou absents dans cette région! Le nombre de personnes originaires du Sud dépend bien sûr totalement de la définition que l'on se donne : natifs du Sud, où qu'ils vivent - il est possible d'en connaître les effectifs, pour ceux qui résident au Soudan et, dans une moindre mesure, pour ceux qui se sont réfugiés dans les pays frontaliers ou descendants de natifs (mais en remontant à quelle profondeur générationnelle ?).

42. L'on ne peut retrancher les $0,6 \mathrm{M}$ tels quels, dans la mesure où une proportion des morts $\mathrm{du}$ fait de la guerre seraient décédés, même en l'absence de cette dernière, durant la période intercensitaire. En appliquant le TNB moyen de 22/1.000 à la population à la mi-période $(6 \mathrm{M})$ et en multipliant par le nombre d'années de la période (10), l'on trouve 1,3 $\mathrm{M}$ de décès, ce qui correspond à $1 / 4$ de la population de départ $(5,3 \mathrm{M}) ; \mathrm{l}$ 'on peut donc compter que, sur les $0,6 \mathrm{M}$ de victimes de la guerre, 150.000 seraient décédées durant la période intercensitaire. Il ne faut donc retrancher que 0,45 M à l'effectif « théorique » de 6,8 M d'originaires du Sud en 1993.

43. Voir MARCHAI. R., art. cit. p. 579.

44. Voir AL-SHAZALI S. al-D., art. cit. p. 161 (chiffres tirés du Steering Committee for the National Dialogue on Peace Settlement and Initiatives, Final report, 1989).

45. Voir dans ce même numéro l'article de Ch. DELMET, «Les relations Nord-Sud au Soudan, 1983-1993».

46. Sur cette dernière région, voir l'article de C. MILLER, « Famine, conflit et aide internationale au Darfour (1990-1992) », dans Égypte/Monde Arabe, n 15/16, pp. 71-96.

47. DE WAAL A., Famine that kills, Clarendon Press, Oxford, 1989, p. 176.

48. On nomme décès "empêchés " des décès qui se seraient produits ultérieurement, dans la même période de référence, si une cause de surmortalité n'était pas intervenue avant. Ici, l'on a compté un TBM de 15/1.000 pour calculer ces décès « empêchés ".

49. Soit $21,14 \mathrm{M}-(400.000-50.000)$. 
50. Voir AL-SHAZALI S. al-D., « Les réfugiés étrangers au Soudan » Égypte/Monde Arabe, n 15-16, pp. 175-190. L'auteur reprend les estimations du COR.

51. Une étude des migrations internes et externes, menée en 1990 dans le Nord-Soudan et par ailleurs assez contestable dans ses méthodes, donne un taux de $3 \%$ de membres des 25.000 familles interrogées présents à l'étranger au moment de l'enquête. Ce taux, appliqué à l'ensemble de la population du Nord-Soudan, donnerait 0,67 $\mathrm{M}$ de Soudanais à l'étranger à cette date (voir Mash al-hijra wa-l quwâ al-'âmila fI-l-sûdàn, vol. 2 (résultats définitifs), Ministère soudanais du Travail et Département de la statistique, OIT, Bureau régional pour les pays arabes, Equipe de travail sur la population et la force de travail au Moyen Orient et dans le Bassin Méditerranéen, Dar al-hijra li-l-nachr, Beyrout, 1991).

52. Est-il besoin de préciser que les évolutions démographiques dont l'image a été esquissée ici devront être régionalisées et désagrégées (au moins selon la dichotomie urbain/rural), travail que l'on s'efforce de mener et dont on rendra compte ultérieurement.

INDEX

Mots-clés : démographie, Soudan

\section{AUTEUR}

FRANÇOIS IRETON

Cedej 